\title{
Multi Group Based Daily Living Activity Recognition (DLAR) using Advanced Machine Learning Algorithm
}

\author{
Doreswamy, Yogesh K M
}

\begin{abstract}
Human activity recognition (HAR) has realized more interest in several research communities given that understanding user activities and behavior help to deliver proactive and personalized services. Different types of noise in wearable sensors data frequently hamper the human activity recognition classification process. Categorically, in this proposed work designs the three-level hierarchical classification structure, i.e., instance based, group and sub-group based and subject based to detect the daily activity of human body motion among activity groups. In correlation with other famous classifiers, for such as Random Forest Tree, J48, Decision Table, Multilayer Perceptron, Nä̈veBayes, oneR and REPTree (Reduced Error Pruning Tree), etc., thorough experiments on the mHealth dataset (Shimmer2 mHealth Data) demonstrate that group based classification achieves the best classification results, reaching RFT 99.97\%. We trained classier in order to estimate accuracy classification based on (gender, age, height, and weight). We applied validation methods to the process, 10-fold cross-validation. For all three classification structure, we achieve high accuracy values for all three classification task.
\end{abstract}

Index Terms: 3D-Accelerometer, Shimmer2, HAR, Wearable Sensor, Machine Learning.

\section{INTRODUCTION}

Physical activity recognition utilizing wearable sensors has empowered established researchers to create novel applications, particularly in the region of social insurance and helped living. As of late, PDAs have been utilized for action acknowledgment, since they are promptly outfitted with a few Sensors helpful for action acknowledgment, for example, movement and area sensors. Additionally, they are conveyed by nearly everybody in their day by day lives [1]. Be that as it may, as far as we could possibly know, there is no examination exploring the presentation of these sensors in detail considering distinctive capabilities, more tasteful, diverse telephone conveying positions, both independently, just as in mix. A few scientists have just explored the blend of different movement sensors in action acknowledgment [2]. For instance, in be that as it may, these past investigations have investigated the blend of different movement sensors just in some specific situations. So as to respond to our inquiry, we think about the job of these sensors in detail in various situations [3]. We completed three diverse

Revised Manuscript Received on July 10, 2019.

Yogesh K M, Computer Science, Mangalore University, Mangalore, Karnataka, India.

Doreswamy, Computer Science, Mangalore University, Mangalore, Karnataka, India. assessment situations so as to cover the most normally utilized situations in the past investigations Specifically client with his/her own information. In addition, we assess the acknowledgment execution with four unique sensors: an accelerometer, a spinner, a direct speeding up and a magnetometer and a gyro meter, a magnetometer [4]. The straight speeding up sensor is a virtual sensor, got from the accelerometer by expelling the gravity part. These sensors are chosen Sensors since they were utilized in past action acknowledgment thinks about [5]. The fundamental center, in any case, is on the accelerometer and the spinner, since these are the for the most part utilized sensors in comparative examinations. Specifically, the objective of this paper is to give an itemized investigation of whether to combine information from numerous sensors. We trust that our exertion will help the readership and this will spare time for future investigations by not rehashing similar analyses.

\section{RELATED WORK}

Human activity recognition wearable sensors is an exceptionally across the board examine subject. Telephone based accelerometers to perform movement acknowledgment, an errand which includes recognizing the physical action a client is performing. To actualize this framework creators gathered named accelerometer information from twenty-nine clients as they performed day by day exercises, for example, strolling, running, climbing stairs, sitting, and standing, and after that amassed this time arrangement information into precedents that condense the client movement more than 10-second interims. Creators at that point utilized the subsequent preparing information to instigate a prescient model for action acknowledgment. Prior work by Kwapisz J R et al [6]. Introduce a pushed forward estimation of delicate biometric data from inertial sensor. By tackling distinctive classification undertakings like age, weight and tallness based on the movement information of human strolling steps spoken to by increasing speeds and rakish speeds. Information were recorded by one sensor set at different areas on the human body, to be specific the chest, the lower back, the wrist and the lower leg. The outcomes demonstrate that these classification errands can be comprehended well by utilizing accelerometers and additionally whirligigs at any of the given order just as given areas. 
The order rates were most astounding for sensors situated at the lower back and chest in every one of the investigations, yet at the same time practically high when the sensor is connected to the wrist or lower leg. The tests have clarified that there isn't one component for the most part in charge of any of the refinements important for a characterization. In any case, the component significance in every one of the grouping gave pointers about what blend of highlights delivers the best outcomes. The most significant endings were that precise speeds showed improvement over increasing velocities. Qaiser Riaz Akker et al [7], earlier work by Incel et al [8]. Studies movement acknowledgment examines utilizing PDAs. In any case, most research depicted in that still includes disconnected handling of the information gathered on the advanced mobile phone. Kunze et al [9]. Examined how increasing speed and spinner signals are influenced by sensor uprooting.

\section{PROPOSED MODEL}

Fig. 1. Proposed Human Activity Recognition (HAR) System

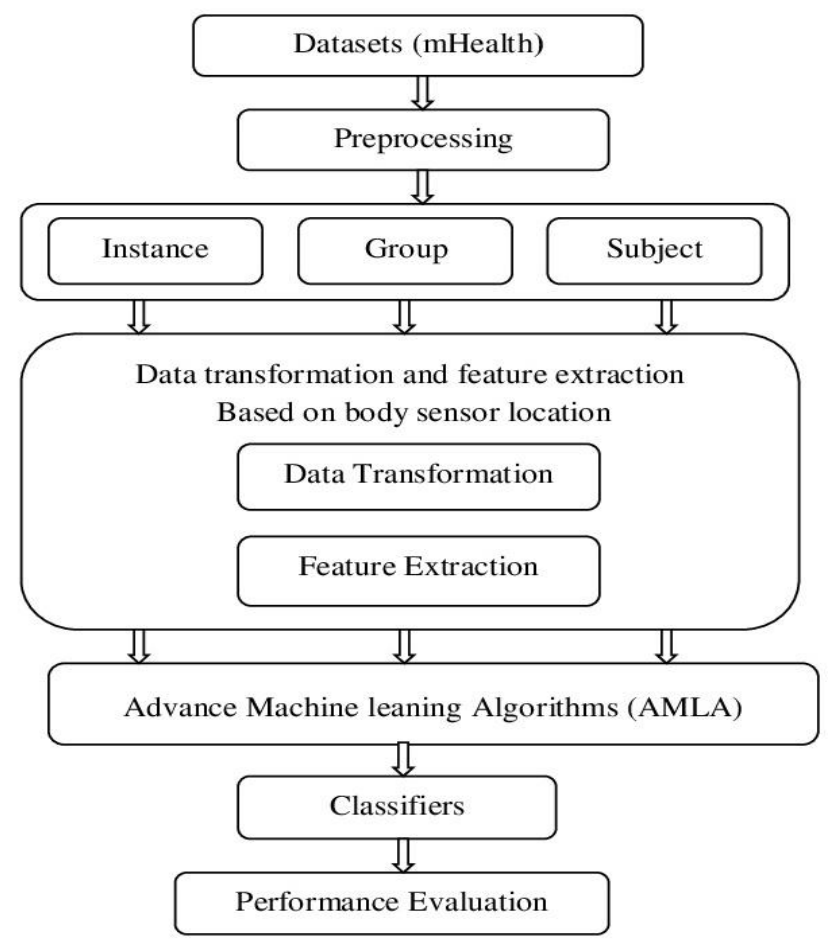

\section{A. Data Set Description}

Characterize information related to propose inquire about examination is MOBILE HEALTH (mHealth) informational collection, which is fundamentally produced by Banos, et.al [1]. what's more, facilitated to AI document of place for Machine Learning (ML) and intelligent systems (IS) at the college of California, Irvine(UCI), This mHealth information is utilized as auxiliary information for learning revelation identified with human movement acknowledgments and pulse exercises rely upon shifting human exercises [1], [10].

\section{B. Data Selection}

Primary information were obtained from three diverse portable wellbeing sensor hubs put at the chest position containing accelerometer and electrocardiogram (ECG) sensors, and a sensor put at the left ankle (LA) and the right-lower-arm (RLA) positions contain an accelerometer, magnetometer and gyro meter. The acceleration(x, y, and $\mathrm{z}$ ), magnetometer(x,y, and $\mathrm{z}$ ) and $\operatorname{gyroscope}(\mathrm{x}, \mathrm{y}$, and $\mathrm{z}$ ) produce three-dimensional information. Furthermore, Electrocardiogram (ECG) sensors produce two dimensional information (L1, L2) individually Lead-I (L1) and Lead-II (L2). In this paper, the chest sensor accelerometer informational indexes are chosen for comprehension about human body movement regarding chest position.

Table1. Activity Set

\begin{tabular}{|c|c|c|c|}
\hline Sl.No & $\begin{array}{c}\text { Human Physical } \\
\text { Activity(HPA) }\end{array}$ & $\begin{array}{c}\text { Actual time } \\
\text { in second }\end{array}$ & Time \\
\hline 1 & Standing still & 61.44 & $1 \mathrm{~min}$ \\
\hline 2 & Sitting and relaxing & 61.44 & $1 \mathrm{~min}$ \\
\hline 3 & Lying down & 61.44 & $1 \mathrm{~min}$ \\
\hline 4 & Walking & 61.44 & $1 \mathrm{~min}$ \\
\hline 5 & Climbing stairs & 61.44 & $1 \mathrm{~min}$ \\
\hline 6 & Waist bends forward & 20 & $20 \mathrm{sec}$ \\
\hline 7 & Front elevation of arms & 20 & $20 \mathrm{sec}$ \\
\hline 8 & Knees bending & 20 & $20 \mathrm{sec}$ \\
\hline 9 & Cycling & 61.44 & $1 \mathrm{~min}$ \\
\hline 10 & Jogging & 61.44 & $1 \mathrm{~min}$ \\
\hline 11 & Running & 61.44 & $1 \mathrm{~min}$ \\
\hline 12 & Jump front \& back & 20 & $20 \mathrm{sec}$ \\
\hline
\end{tabular}

\section{Data Preprocessing}

The raw accelerometer sensor information regularly needs to drawing closer predisposed in order to dispose of filthy, uproarious what's more, excess information. As these sorts of information are free from uproarious, in this way, information changes are proposed for lessening the bigger size of the information to the diminished portrayal by creating highlights for a changing window estimate.

\section{Data Transformation and Feature Generation}

MHealth information contains 10 people/clients of varying gender, height, weight and different age and each user was done 12 exercises that are appeared table1. The mHealth information contains 12, 15,749 occurrences. All detecting modalities are recorded at an examining rate of $50 \mathrm{~Hz}$, the sensor limit gadget was sufficient for getting Human Physical Activity (HPA). Time taken for producing one occasion was $0.02 / \mathrm{ms}$ time, which is relating to $\mathrm{t}=(1 / 50) \mathrm{Hz}$ $=0.02 / \mathrm{ms}$. Each client played out all exercises and every action was performed for $1 \mathrm{~min}$ and the exercises midriff twist forward, front height of arms, knee bowing and hop front and back were performed $20 / \mathrm{sec}$ as it were. For example, time taken for every action can be determined utilizing the underneath recipe [1].

$$
T=T_{A} \frac{E_{t} * I_{t}}{t}
$$

$T_{A}=$ Total time taken for each activity

$E_{t}=$ Time take for each instance

$I_{t}=$ total number of instance in an individual activity

$\mathrm{T}=$ Time in second 
Ordinary investigation of classification methodology could be unequivocally reasonable to time succession increasing speed sensor information, however, it leads computational multifaceted nature and decreases proposition of a more tasteful. Consequently, information change is sent on the crude time arrangement window tests. To achieve this information is partitioned into changing windows sizes that are relating to $10,20,30,40,50$, and 60-second, information part containing 512,1024,1536, 2048, 2560 and 3072, readings separately Table 1 .

\section{E. Feature Extraction}

An occurrence of wt seconds ( $\mathrm{W}=\mathrm{fs}$ wt samples) is utilized to compute the list of capabilities for a specific action. Here, fs are the recurrence testing of the speeding up information. For each element of the quickening sensor, twelve distinct highlights in the time-space are separated. Following is the detail of each time space includes. Let $x$ is the sign of window measure wt seconds having $\mathrm{W}$ windows tests or information focuses [11]. . Presents a total rundown of highlights extricated from various parts of increasing velocities and rakish speeds. For every single step, the list of capabilities comprises of includes altogether. Measurable highlights include: mean, middle, worldwide least, worldwide most extreme, first Quartile, third Quartile, standard deviation, mean an outright mistake, mean square blunder and root mean square the majority of the rest of the features are figured for all 3D increasing velocities.

Table 2

Description of the Extracted Features the Time and Frequency Domain is computed

\begin{tabular}{|c|c|c|c|}
\hline Features Name & Sensor & Axis & Total \\
\hline Mean & A & $\mathrm{X}, \mathrm{Y}, \mathrm{Z}$ & 9 \\
\hline Median & A & $\mathrm{X}, \mathrm{Y}, \mathrm{Z}$ & 9 \\
\hline Minimum & A & $\mathrm{X}, \mathrm{Y}, \mathrm{Z}$ & 9 \\
\hline Maximum & A & $\mathrm{X}, \mathrm{Y}, \mathrm{Z}$ & 9 \\
\hline First Quartile Range & $\mathrm{A}$ & $\mathrm{X}, \mathrm{Y}, \mathrm{Z}$ & 9 \\
\hline Entropy & $\mathrm{A}$ & $\mathrm{X}, \mathrm{Y}, \mathrm{Z}$ & 9 \\
\hline Standard Deviation & $\mathrm{A}$ & $\mathrm{X}, \mathrm{Y}, \mathrm{Z}$ & 9 \\
\hline Mean Absolute Value & $\mathrm{A}$ & $\mathrm{X}, \mathrm{Y}, \mathrm{Z}$ & 9 \\
\hline Harmonic Mean & $\mathrm{A}$ & $\mathrm{X}, \mathrm{Y}, \mathrm{Z}$ & 9 \\
\hline Variance (VR) & $\mathrm{A}$ & $\mathrm{X}, \mathrm{Y}, \mathrm{Z}$ & 9 \\
\hline Root Mean Square & $\mathrm{A}$ & $\mathrm{X}, \mathrm{Y}, \mathrm{Z}$ & 9 \\
\hline Skewness & $\mathrm{A}$ & $\mathrm{X}, \mathrm{Y}, \mathrm{Z}$ & 9 \\
\hline Kurtosis & $\mathrm{A}$ & $\mathrm{X}, \mathrm{Y}, \mathrm{Z}$ & 9 \\
\hline Simple Squared Integral & $\mathrm{A}$ & $\mathrm{X}, \mathrm{Y}, \mathrm{Z}$ & 9 \\
\hline
\end{tabular}

MHealth information contains 10 people of shifting age, stature, weight and diverse sexual orientation and every client was completed 12 various sorts of movement that are appeared in table 2. The component was extricated diving window wt of 10/sec time term [6]. Shimmer2 wearable information in $\mathrm{X}, \mathrm{Y}$ and $\mathrm{Z}$ measurements of the three significant body position and the significant highlights are extricated and got from every 512 occasions in each physical action this window to perceive the development as being one of twelve possible choices. The sampler recurrence was recorded the accelerometer information at the $50 \mathrm{~Hz}$ limit. An association of one $1 / \mathrm{sec}$ is considered for sliding the window on the shimmer2 sensor information [12]. Time-space highlights are proposed on the span of $10 / \mathrm{sec}$ and 512 occasions are gotten in every action from each put of accelerometer sensors, set on the Chest, Right Lower Arms (RLA) and Left Ankle(LA), separately. Table 3 demonstrates the scattering of examples of the distinctive day by day physical exercises in the component sets [13].

\section{Fig2. Schematic representation of Advanced Data Mining Techniques}

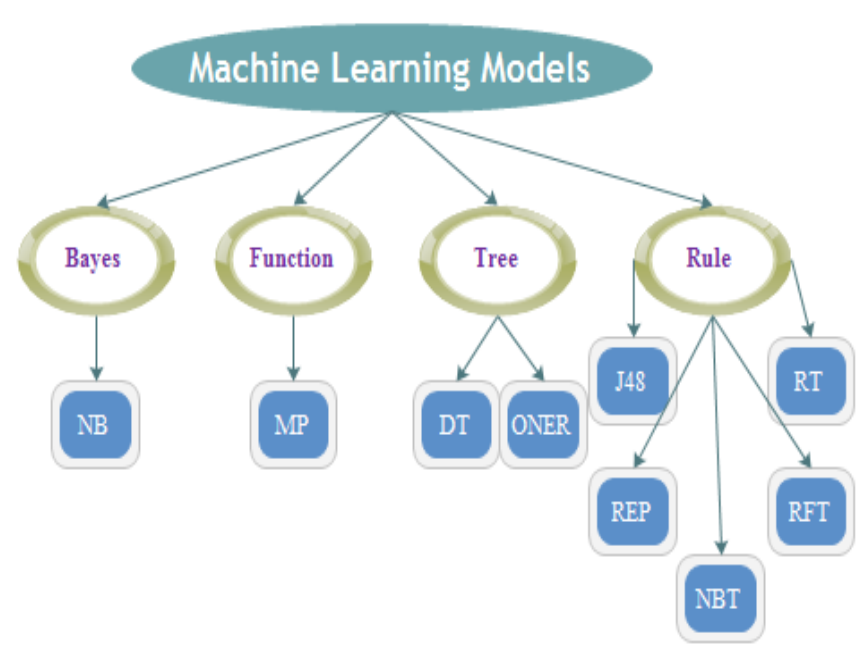

IV. FEATURE GENERATION

Classification is the way toward structure a model (or capacities), which depicts and recognizes information classes or ideas, for the reasons for having the option to utilize the model to anticipate the class of articles whose class name is obscure. The characterization models are built dependent on the investigation of 3/4thh of preparing information whose class mark is known and tried on $1 /$ fourth of real cases as testing tests. The propelled Data Mining Techniques sent for grouping of mHealth informational collections are classified in table3 [14].

Table 3

Number of Features Vectors per User and Activities

\begin{tabular}{|c|c|c|c|c|c|c|c|c|c|c|c|}
\hline \multirow{2}{*}{ Nor } & \multicolumn{10}{|c|}{ Person } & \multirow{2}{*}{ Total } \\
\cline { 2 - 10 } $\mathbf{1}$ & 6 & 6 & 6 & 6 & 6 & 6 & 6 & 6 & 6 & 6 & $\mathbf{6 0}$ \\
\hline $\mathbf{2}$ & 6 & 6 & 6 & 6 & 6 & 6 & 6 & 6 & 6 & 6 & $\mathbf{6 0}$ \\
\hline $\mathbf{3}$ & 6 & 6 & 6 & 6 & 6 & 6 & 6 & 6 & 6 & 6 & $\mathbf{6 0}$ \\
\hline $\mathbf{4}$ & 6 & 6 & 6 & 6 & 6 & 6 & 6 & 6 & 6 & 6 & $\mathbf{6 0}$ \\
\hline $\mathbf{5}$ & 6 & 6 & 6 & 6 & 6 & 6 & 6 & 6 & 6 & 6 & $\mathbf{6 0}$ \\
\hline $\mathbf{6}$ & 2 & 2 & 2 & 2 & 2 & 2 & 2 & 2 & 2 & 2 & $\mathbf{6 0}$ \\
\hline $\mathbf{7}$ & 2 & 2 & 2 & 2 & 2 & 2 & 2 & 2 & 2 & 2 & $\mathbf{6 0}$ \\
\hline $\mathbf{8}$ & 2 & 2 & 2 & 2 & 2 & 2 & 2 & 2 & 2 & 2 & $\mathbf{6 0}$ \\
\hline $\mathbf{9}$ & 6 & 6 & 6 & 6 & 6 & 6 & 6 & 6 & 6 & 6 & $\mathbf{6 0}$ \\
\hline $\mathbf{1 0}$ & 6 & 6 & 6 & 6 & 6 & 6 & 6 & 6 & 6 & 6 & $\mathbf{6 0}$ \\
\hline $\mathbf{1 1}$ & 6 & 6 & 6 & 6 & 6 & 6 & 6 & 6 & 6 & 6 & $\mathbf{6 0}$ \\
\hline $\mathbf{1 2}$ & 2 & 2 & 2 & 2 & 2 & 2 & 2 & 2 & 2 & 2 & $\mathbf{6 0}$ \\
\hline All & $\mathbf{5 6}$ & $\mathbf{5 6}$ & $\mathbf{5 6}$ & $\mathbf{5 6}$ & $\mathbf{5 6}$ & $\mathbf{5 6}$ & $\mathbf{5 6}$ & $\mathbf{5 6}$ & $\mathbf{5 6}$ & $\mathbf{5 6}$ & $\mathbf{5 6 0}$ \\
\hline
\end{tabular}

Human action recognition frameworks depend on cutting edge information mining calculations to anticipate a person's action amid a specific timescale. 
Moreover, it has been stressed that distinctive classification techniques could be utilized in HAR frameworks, contingent upon the specific qualities of every situation (e.g., the arrangement of exercises, the kind of sensors, etc). Miguel A. Labrador Oscar D.et al [12]. Expand on the benefits of actualizing a totally portable HAR framework as far as dependability, adaptability, and vitality utilization, just to specify a couple. Yet, such an errand involves the assessment of a classification model on the Saxophone, which achieves an

\begin{tabular}{|c|c|}
\hline Features & Description \\
\hline Mean & The DC component (average value) of the signal over the window \\
\hline Standard Deviation & Measure of the spreadness of the signal over the window \\
\hline Inter Quartile Range & The median of the upper half of the data set. \\
\hline Mean Absolute Value & The summation inters quartile range which describes the variability of a window. \\
\hline Harmonic Mean & The harmonic mean is the reciprocal of the arithmetic mean of the reciprocals. \\
\hline Variance & The square of standard deviation \\
\hline Root Mean Square & The quadratic mean value of the signal over the window \\
\hline Skewness & The degree of asymmetry of the sensor signal distribution \\
\hline kurtosis & The degree of peakedness of the sensor signal distribution \\
\hline Simple Squared & The basic squared fundamental computes the efficiencies of signs \\
\hline Integral & The median signal value over the window \\
\hline Median & The minimum value over the window \\
\hline Maximum & The maximum values over the window \\
\hline First Quartile Range & Measure of the statistical dispersion, 25th percentiles of the signal over the window \\
\hline Third Quartile Range & Measure of the statistical dispersion, 75th percentiles of the signal over the window \\
\hline
\end{tabular}

\section{A. Evaluation}

This stage includes thinking about different models and picking the best one dependent on their prescient presentation (i.e., clarifying the changeability being referred to and creating stable outcomes crosswise over examples). This may seem like a basic task, yet in reality, it some of the time includes an exceptionally intricate procedure. There are assortments of systems created to accomplish that objective numerous of which depend on purported "aggressive assessment of models," that is, applying various models to similar information set and after that contrasting their exhibition with pick the best [16].

1) True Positives (TP): The number of positive instances that are classified as positive.

2) True Negatives (TN): The number of negative instances that are classified as negative.

3) False Positives (FP): The number of negative instances that are classified as positive.

4) False Negatives (FN): The number of positive instances that are classified as negative.

Accuracy: It is the most standard metric to summarize the overall classification performance for all classes and it is dened as follows:

$$
\text { Accuracy }=\frac{(\mathrm{TP}+\mathrm{TN})}{(\mathrm{TP}+\mathrm{TN}+\mathrm{FP}+\mathrm{FN})}
$$

Precision: It is often referred to as positive predictive value and is the ratio of correctly classified positive instances to the total number of instances classified as positive extra test that is actualizing every single more tasteful under the Android stage. This could be very tedious given the hidden unpredictability in the usage of AI calculations, alongside the computational limitations present in cell phones. The focal point of this paper, in this manner, is to use the usage of various classification strategies given by WEKA to empower Classifiers assessment so as to assemble these under the Android structure [15].

\section{Description}


Further the diverse propelled classifiers strategies such as Nave bayes, multilayer perceptron, choice Table, OneR, J48, Random Forest, Random Tree, and Reduced Error Pruning (REP) Tree and gullible Bayes Tree were tested and assessed on three classifications of information such case, gathering and subject based information and separately called as Instance based order (IBD), Group based arrangement (GBC) and Subject based order (SBC).The trial consequences of everyone is portrayed in the accompanying segment [7].

\section{A. Group and Sub-Group based classification}

Be that as it may, in this paper bunch based grouping we expect that the application space enables us to use from the earlier information that the entire gathering of marked examples have a place to the class, and furthermore yet known, class [7]. Along these lines, we just need consider the 12 conceivable class marks for the arrangement. Group Based Classification (GBC) there four diverse characterization errands. Preparing

\begin{tabular}{lc}
\hline Algorithm 1 Classifier for Gender based data & Algorithm 1 Classifier for Gender based data \\
\hline INPUT: Gender based data values & INPUT: Gender based data values \\
INPUT: Gender based classification accuracy & INPUT: Gender based classification accuracy \\
Classification $($ gender, classifiers $)$ & Classification $($ gender, classifiers $)$ \\
\{ & $\{\quad$ if $($ gender $==N U L L)$ \\
if $($ gender $==N U L L)$ & Classifiers $($ exit $)$ \\
Classifiers $($ exit $)$ & else classification $($ gender $\rightarrow$ classifiers $)$ \\
else classification $($ gender $\rightarrow$ classifiers $)$ & \} \\
\} &
\end{tabular}

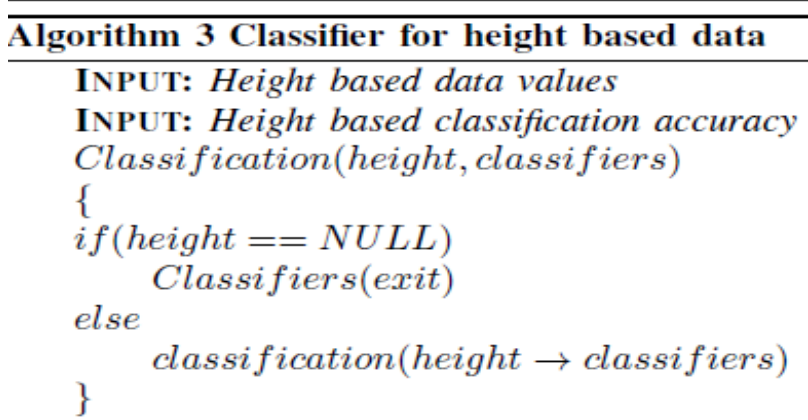

and approval information were likewise arranged for classification inside member subgroups for sexual orientation, weight stature and age Classification. In table the qualities of the populace inside various classification undertakings are exhibited as appeared table 4. The above table shows four diverse grouping errands. Sex arrangement, in this characterization seven male and three female, age order have five more prominent than or equivalent to 25 age and under 25

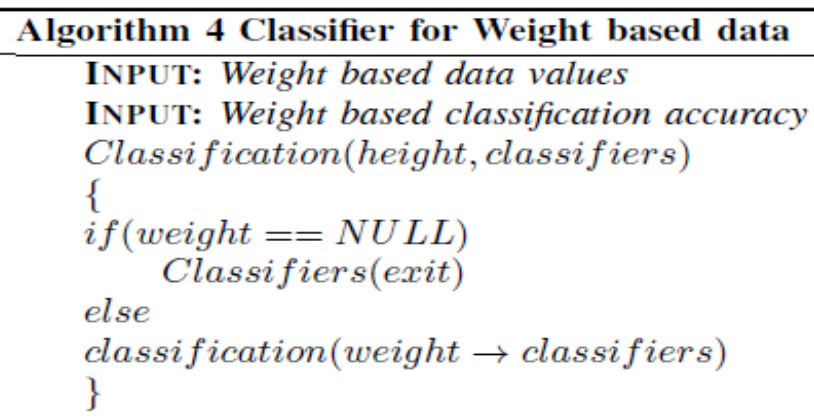

age, stature arrangement have three more noteworthy than or equivalent to $170 / \mathrm{cm}$ stature and under $170 / \mathrm{cm}$ tallness. Also weight arrangement has five more prominent than or equivalent to $25 / \mathrm{kg}$ weight $/ \mathrm{kg}$ and under $25 / \mathrm{kg}$. In any case, the focal point of this paper is on consecutive compound choices and bunch based order specifically table5. identified by movement chronicles of any of the utilized sensors. The aftereffects of the ternary classification for accelerometer sensor on chest position sensor are given in table 7 .

Table 5: Number of Features Vectors per User and Activities

\begin{tabular}{|c|c|c|c|c|c|c|c|c|c|}
\hline Group & DT & J48 & MP & NB & NBT & OneR & RFT & RT & REP \\
\hline Male & 70.79 & 83.33 & 65.26 & 65.02 & 79.21 & 53.97 & 99.96 & 99.96 & 79.75 \\
\hline Female & 71.87 & 84.39 & 68.04 & 66.12 & 79.37 & 56.72 & 99.96 & 99.96 & 79.9 \\
\hline A > = 26 & 71.42 & 84.38 & 67.04 & 65.51 & 8016 & 55.06 & 99.95 & 99.96 & 80.74 \\
\hline $\mathbf{A}<\mathbf{2 6}$ & 70.66 & 82.55 & 64.68 & 65.11 & 77.89 & 54.4 & 99.97 & 99.97 & 78.38 \\
\hline H > 172 & 70.52 & 82.93 & 64.7 & 65.09 & 78.63 & 54.06 & 99.97 & 99.97 & 79.33 \\
\hline H $<\mathbf{1 7 2}$ & 72.01 & 84.71 & 68.19 & 65.73 & 80.2 & 55.9 & 99.95 & 99.95 & 80.49 \\
\hline $\mathbf{W}>\mathbf{7 0}$ & 71.37 & 83.81 & 66.45 & 65.53 & 79.95 & 54.3 & 99.96 & 99.96 & 80.66 \\
\hline $\mathbf{W}<\mathbf{7 0}$ & 70.86 & 83.48 & 65.73 & 65.17 & 78.56 & 55.28 & 99.96 & 99.97 & 78.93 \\
\hline
\end{tabular}

\section{Gender Based Classification (GBC):}

Our objective was to demonstrate that classification errands with respect to the sex order of the preliminary subject can be performed adequately well by utilizing the proposed sensors connected to chest position of human. The sex can be identified by movement chronicles of any of the utilized sensors the outcomes introduced in table 6 .

\section{Age Based Classification (ABC):}

Here, the Classification assessed the task to two classes as indicated by two age gatherings (age $>=26$, age $<26$ ) of members. 


\section{Height Based Classification (HBC):}

Another objective was body height classification from just increasing velocities sensor. The age gathering of people can be identified by movement chronicles of shimmer2 sensor gadget. The consequences of the ternary classification for accelerometer sensor on chest position sensor are given in table 8. Here, the Classification assessed the task to two classes as indicated by two age gatherings (tallness $>=172$, stature > 172) of members.

\section{Weight Based Classification (WBC):}

Another objective was body weight classification from just increasing speeds sensor from chest position. The weight gathering of people can be identified by movement accounts of shimmer2 sensors. The consequences of the ternary Classification for accelerometer sensor on chest position sensor are given in table 8 . Here, the Classification evaluated the task to two classes as per two weight gatherings (weight > $=70$, weight $>70$ ) of members.

\section{A. Instance Based Classification}

Examinations of various classifiers are done on every window containing number of occasions: W1-512, W2-1024, W3-1536, W4-2048, W5-2560, and W6-3072 in every action regarding all matters. The normal grouping exactness of each model on every one of the 10 people under every window measure is figured. A similar methodology is registered utilizing calculation given underneath. The normal perforce of the classifiers on changing breeze measure is shifting. Be that as it may, the RFT and RT classifiers are going $100 \%$ classification exactness. This table 4 determines the prescient precision related with every one of the order strategies for each case in every one of the windows [13].

Table 6: Group and sub-group based classification

\begin{tabular}{|c|c|c|c|c|c|c|c|c|c|}
\hline Instance & DT & J48 & MP & NB & NBT & $\begin{array}{c}\text { One } \\
\text { R }\end{array}$ & RFT & RT & REP \\
\hline W1:512 & 72.1 & 83.4 & 68.2 & 66.5 & 77 & 57.3 & 100 & 99 & 77.2 \\
\hline W1:1024 & 72 & 82.4 & 64.7 & 66.8 & 76.8 & 56.7 & 100 & 99 & 77.2 \\
\hline W1:1536 & 74.5 & 83.2 & 67.6 & 70.9 & 79.3 & 59.4 & 100 & 99 & 79.7 \\
\hline W1:2048 & 74 & 82.7 & 71.6 & 70.5 & 78.2 & 59.1 & 100 & 99 & 79.7 \\
\hline W1:2560 & 73.6 & 82.4 & 68.6 & 70.1 & 78.1 & 58.8 & 100 & 99 & 78.8 \\
\hline W1:3072 & 73.2 & 82 & 69.8 & 69.7 & 77.1 & 58.5 & 100 & 99 & 78.8 \\
\hline
\end{tabular}

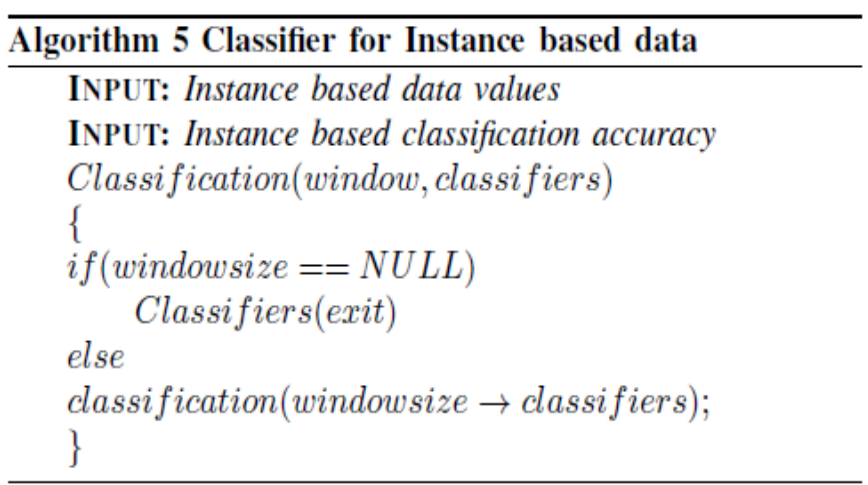

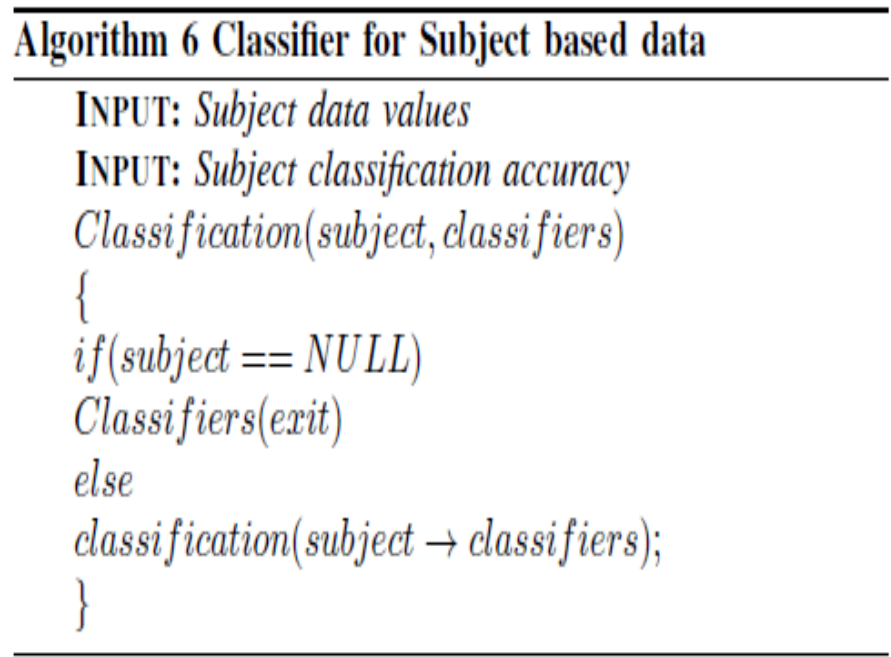

Fig3. Group and sub-group based classification

Group and sub-group Based Classification Accuracy

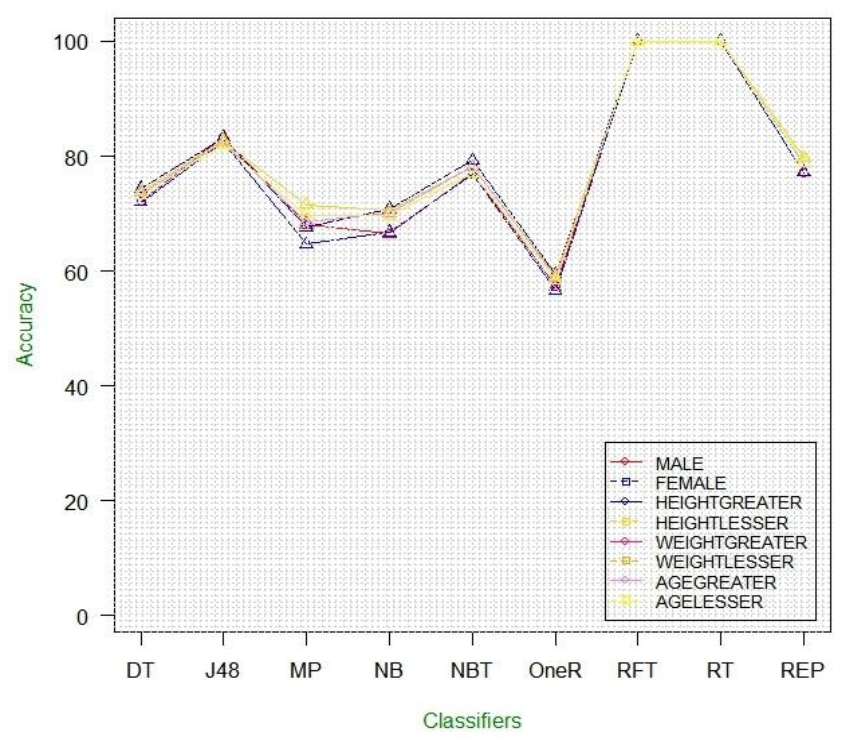

Fig: 4 Window based classification

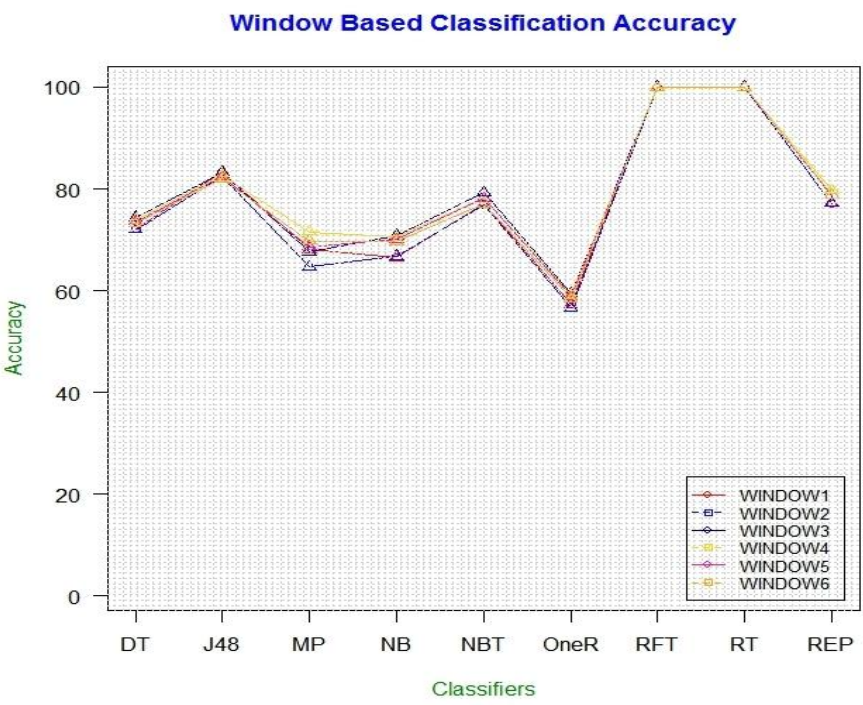




\section{B. Subject Based Classification}

This step was performed by extracting the raw accelerometer data from subject or person wise during the data collection process. The each person took all twelve activities. The person was taken $1 / \mathrm{min}$ time for each activity, and each data, and extracted features for every 10 to $60 / \mathrm{s}$ data and apply advance classifiers methods. At the human chest position 12, 15,749 instances were produced, with 3072 instances for each activity, except Waist bends forward, Front elevation of arms, Knees bending, and jump front and back, where the trainings for both instance were conducted using the 10 fold cross validation method. Tastings Were then performed using the untrained datasets to test the accuracy of the trained classifiers, here we showed final average of all nine advanced classifiers methods of all ten person. In this methods Random forest tree (RFT) and Random tree (RT) gives more than $99 \%$ of accuracy and also lying down and activity data can be divide 10/s, 20/s, 30/s, 40/s, 50/s, 60/s

standing still activity getting more than $95 \%$ of accuracy because there is no lot of difference between lying down, sitting and relaxing and standing still so both activity got more than $99 \%$ accuracy in all selected advanced classifiers. Here we have showed two different graphical representations figure 5 shows Column representation and figure 6 shows 2-D line graphical representation [13].

\section{Table 7: Subject Based Classification}

\begin{tabular}{|c|c|c|c|c|c|c|c|c|}
\hline Id & DT & J48 & MP & NB & NBT & OneR & RFT & REP \\
\hline P1 & 72.4 & 82.5 & 67.6 & 67.1 & 77.7 & 56.8 & $\mathbf{9 9 . 7}$ & 78.3 \\
\hline P2 & 69.6 & 81.3 & 66.6 & 65.4 & 76.5 & 54.6 & $\mathbf{9 9 . 9}$ & 78.8 \\
\hline P3 & 70.5 & 82.9 & 62.0 & 64.2 & 78.3 & 52.2 & $\mathbf{9 9 . 7}$ & 78.2 \\
\hline P4 & 68.1 & 81.2 & 62.4 & 63.5 & 76.3 & 54.0 & $\mathbf{9 9 . 7}$ & 76.7 \\
\hline P5 & 68.2 & 83.7 & 64.5 & 61.9 & 76.8 & 55.6 & $\mathbf{9 9 . 6}$ & 78.2 \\
\hline P6 & 72.4 & 85.6 & 68.6 & 64.5 & 82.6 & 53.4 & $\mathbf{9 9 . 2}$ & 82.2 \\
\hline P7 & 71.5 & 83.3 & 66.5 & 65.7 & 79.1 & 54.4 & $\mathbf{9 9 . 7}$ & 80.3 \\
\hline P8 & 74.9 & 86.8 & 71.9 & 69.2 & 83.6 & 57.6 & $\mathbf{9 9 . 6}$ & 83.1 \\
\hline P9 & 73.4 & 87.3 & 68.6 & 69.02 & 85.1 & 56.1 & $\mathbf{9 9 . 8}$ & 84.0 \\
\hline P10 & 69.8 & 81.3 & 61.7 & 62.8 & 76.2 & 52.8 & $\mathbf{9 9 . 4}$ & 77.8 \\
\hline
\end{tabular}

Fig: 5 Final classification accuracy

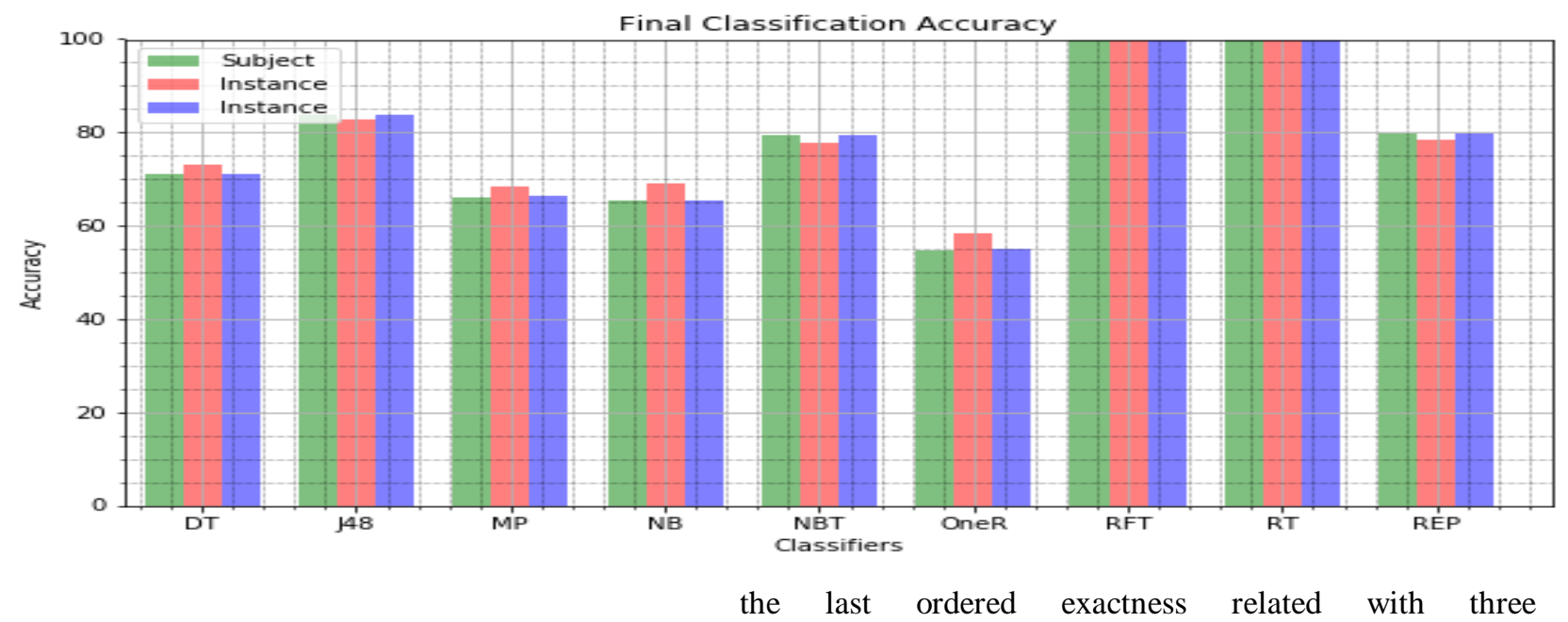

The figures demonstrate that in most cases we can achieve high levels of accuracy. For the three most common activities, lying, standing still, and sitting and relaxing. We generally achieve accuracies above $90 \%$. Sitting and relaxing appears easier to identify than standing still, which seems to make sense, since jogging involves more extreme changes in acceleration. It appears much more difficult to identify the two stair climbing activities, but as we shall see shortly, that is because those two similar activities are often confused with one another. Note that although there are very few examples of sitting and standing, we can still identify these activities quite well, because, as noted earlier, the two activities cause the device to change orientation and this is easily detected from the accelerometer data. Our results indicate that none of the three learning algorithms consistently performs best, but the multilayer perceptron does perform best overall. More detailed results are presented in above Tables, which show the activity accuracy with each of the learning algorithms [17].

\section{CLASSIFICATION ACCURACY AND RESULT}

The outline results for human action acknowledgment experiments are introduced in Table 11. This table indicates characterizations

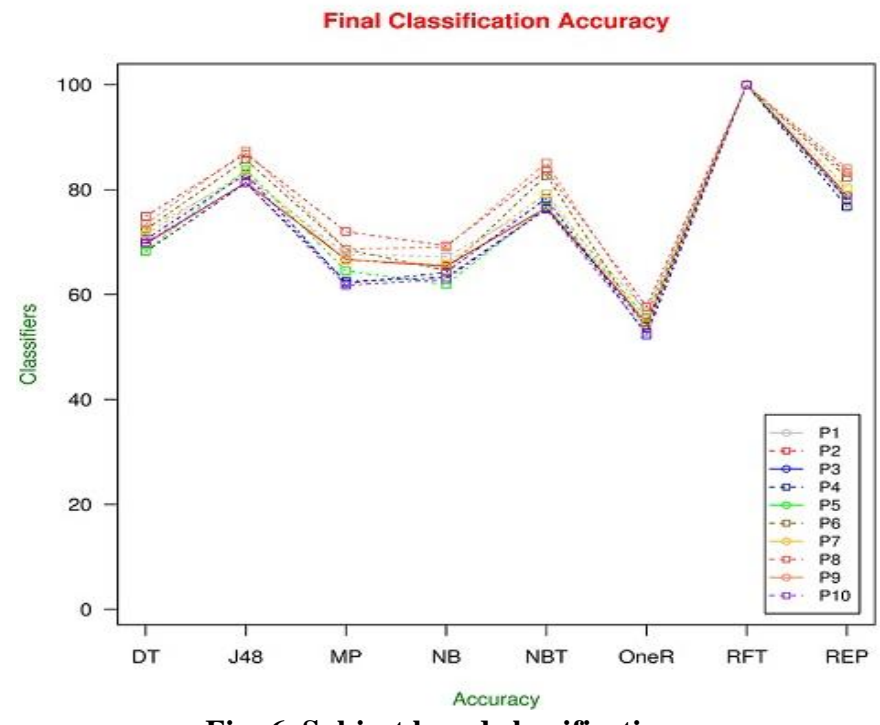

Fig: 6. Subject based classification 
The J48 Decision tree classifier pursues the accompanying straightforward calculation. So as to characterize another thing, it first needs to make a choice tree dependent on the trait estimations of the accessible preparing information. While evaluating the general execution of the classifier (i.e., the last line of Table 11), in this general outline Random woodland tree(RFT) and irregular tree(RT) classifiers gives more than $99 \%$ precision in every one of the three sorts of order [18]. For grouping issues, given a lot of straightforward trees and a lot of arbitrary indicator factors, the Random Forest strategy characterizes an edge work that measures the degree to which the normal number of votes in favor of the right class surpasses the normal vote in favor of some other class present in the reliant variable. This measure gives us not just with an advantageous method for making forecasts, however likewise

with a method for partner a certainty measure with those forecasts. Beneath tables contain by and large exactness from accelerometer chest sensor information [19].

\section{CONCLUSION}

The work described in this paper is part of a larger effort to mine sensor data from shimmer wireless sensor devices. We plan to continue mHealthDroid-based project, applying the accelerometer data to other tasks besides activity recognition and collecting and mining other sensor data, especially Accelerometer, magnetometer, and gyro meter data. We believe that shimmer2 (BUR10) sensor data provides tremendous opportunities for data mining and we intend to leverage mHealthDroid-based data collection/data mining platform to the fullest extent possible.

\section{REFERENCES}

1. Banos, O., Garcia, R., Holgado-Terriza, J.A., Damas, M., Pomares, H., Rojas, I., Saez, A., Villalonga, C.: mhealthdroid: a novel framework for agile development of mobile health applications. In: International Workshop on Ambient Assisted Living, Springer (2014) 91-98

2. A. M. Khan, Y.-K. Lee, S. Y. Lee, and T.-S. Kim, "A triaxial accelerometer-based physical-activity recognition via augmented-signal features and a hierarchical recognizer," IEEE transactions on information technology in biomedicine, vol. 14, no. 5, pp. 1166-1172, 2010.

3. D. Trong Bui, N. Nguyen, and G.-M. Jeong, "A robust step detection algorithm and walking distance estimation based on daily wrist activity recognition using a smart band," Sensors, vol. 18, no. 7, p. 2034, 2018.

4. N. D. Nguyen, P. H. Truong, and G.-M. Jeong, "Daily wrist activity classification using a smart band," Physiological measurement, vol. 38, no. 9, p. L10, 2017.

5. N. D. Nguyen, D. T. Bui, P. H. Truong, and G.-M. Jeong, "Classification of five ambulatory activities regarding stair and incline walking using smart shoes,” IEEE Sensors Journal, vol. 18, no. 13, pp. 5422-5428, 2018

6. J. R. Kwapisz, G. M. Weiss, and S. A. Moore, "Activity recognition using cell phone accelerometers," ACM SigKDD Explorations Newsletter, vol. 12, no. 2, pp. 74-82, 2011.

7. Q. Riaz, A. V"ogele, B. Kr"uger, and A. Weber, "One small step for a man: Estimation of gender, age and height from recordings of one step by a single inertial sensor," Sensors, vol. 15, no. 12, pp. 31 999-32 019, 2015.

8. O. D. Incel, M. Kose, and C. Ersoy, "A review and taxonomy of activity recognition on mobile phones," BioNanoScience, vol. 3, no. 2, pp. 145-171, 2013.

9. L. Atallah, B. P. L. Lo, R. C. King, and G.-Z. Yang, "Sensor positioning for activity recognition using wearable accelerometers," IEEE

10. Transactions on Biomedical Circuits and Systems, vol. 5, pp. 320-329, 2011.

11. Ba nos, M. Damas, H. Pomares, I. Rojas, M. A. T'oth, and O. Amft, "A benchmark dataset to evaluate sensor displacement in activity recognition," in Proceedings of the 2012 ACM Conference on Ubiquitous Computing. ACM, 2012, pp. 1026-1035.

12. J. P“arkk"a, M. Ermes, P. Korpip“a”a, J. M“antyj"arvi, J. Peltola, and I. Korhonen, "Activity classification using realistic data from wearable vol. 10, pp. 119-128, 2006

13. O. D. Lara and M. A. Labrador, "A survey on human activity recognition using wearable sensors." IEEE Communications Surveys and Tutorials, vol. 15, no. 3, pp. 1192-1209, 2013.

14. K. Yogesh et al., "Instance based human physical activity (hpa) recognition using shimmer2 wearable sensor data sets," in Advances in Computing, Communications and Informatics (ICACCI), 2017 International Conference on. IEEE, 2017, pp. 995-999.

15. F. Attal, S. Mohammed, M. Dedabrishvili, F. Chamroukhi, L. Oukhellou, and Y. Amirat, "Physical human activity recognition using wearable sensors," Sensors, vol. 15, no. 12, pp. 31 314-31 338, 2015.

16. M. A. Labrador and O. D. L. Yejas, Human activity recognition: Using wearable sensors and smartphones. Chapman and Hall/CRC, 2013.

17. R. Dama`sevi cius, M. Vasiljevas, J. `Salkevi`cius, and M. Wo'zniak, "Human activity recognition in aal environments using random projections," Computational and mathematical methods in medicine, vol. 2016, 2016

18. A. Bayat, M. Pomplun, and D. A. Tran, "A study on human activity recognition using accelerometer data from smartphones," Procedia Computer Science, vol. 34, pp. 450-457, 2014.

19. T. R. Patil and S. Sherekar, "Performance analysis of naive bayes and j48 classification algorithm for data classification," International journal of computer science and applications, vol. 6, no. 2, pp. 256-261, 2013.

20. C. Torres-Huitzil and A. Alvarez-Landero, "Accelerometer-based human activity recognition in smartphones for healthcare services," in Mobile Health. Springer, 2015, pp. 147-169.

\section{AUTHORS PROFILE}

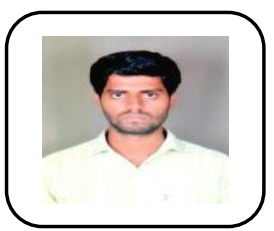

Yogesh K M is currently a Research Scholar department of Computer Science. He received B.Sc. from University of Mysore in 2012 and M.Sc degree in Computer Science from Mangalore University in 2014 respectively. After completion of his Post Graduation Degree. He joined as Research in Computer Science at Mangalore University in the year 2015 still him pursuing $\mathrm{PhD}$ on Human physical activity recognition using mHealth datasets. He has published more that 6 contribute research papers at National/International Journals and Conferences.

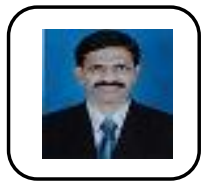

Dr. Doreswamy is currently a Professor of Computer Science in the Department of Computer Science. He received B.Sc. and M.Sc. degree in Computer Science from University of Mysore in 1993 and 1995 respectively. After completion of his Post Graduation Degree. He joined as Associate Professor in Computer Science at Mangalore University in the year 2003. He was the Chairman of the Department of Post-Graduate Studies and Research in Computer Science during 2003-2005 and 2008-2012 and served at various capacities in Mangalore University, as a Chairman and member of DOC, DOS and UG/PG BOS and BOE in Computer Science. Started Ph.D. programme in Computer Science and Technology in Mangalore University with effect from the academic year 2003-04 onwards. His areas of research interests include Data Mining and Knowledge Discovery, Artificial Intelligence, Machine learning and Scalable Advanced Data Mining Algorithms. He has published more that 60 contributed peer-reviewed research papers at National/International Journals and Conferences. He has chaired many National and International Conferences in India. He has completed one minor research project that was sanctioned by University Grants Commission (UGC). He completed Major Research Project entitled "Scientific Knowledge Discovery Systems (SKDS) for advanced Engineering Materials Design Applications "from the funding Agency University Grant Commission, New Delhi, INDIA 